\title{
Underlying Thermodynamics of pH-Dependent Allostery
}

\author{
Natali V. Di Russo, ${ }^{\dagger}$ Marcelo A. Martí, ${ }^{\dagger}$ and Adrian E. Roitberg ${ }^{*} \dagger$ \\ ${ }^{\dagger}$ Quantum Theory Project and Department of Chemistry, University of Florida, Gainesville, Florida 32611, United States \\ ‡Departamento de Química Inorgánica, Analítica y Química Física/INQUIMAE-CONICET and Departamento de Química \\ Biológica, Facultad de Ciencias Exactas y Naturales, Universidad de Buenos Aires, Buenos Aires C1428EGA, Argentina
}

\section{Supporting Information}

ABSTRACT: Understanding the effects of coupling protein protonation and conformational states is critical to the development of drugs targeting $\mathrm{pH}$ sensors and to the rational engineering of $\mathrm{pH}$ switches. In this work, we address this issue by performing a comprehensive study of the $\mathrm{pH}$-regulated switch from the closed to the open conformation in nitrophorin 4 (NP4) that determines its $\mathrm{pH}$-dependent activity. Our calculations show that D30 is the only amino acid that has two significantly different $\mathrm{p} K_{\mathrm{a}} \mathrm{s}$ in the open and closed conformations, confirming its critical role in regulating $\mathrm{pH}$ dependent behavior. In addition, we describe the free-energy landscape of the conformational change as a function of $\mathrm{pH}$, obtaining accurate estimations of free-energy barriers and equilibrium constants using different methods. The underlying thermodynamic model of the switch workings suggests the possibility of tuning the observed $\mathrm{p} K_{\mathrm{a}}$ only through the conformational equilibria, keeping the same conformation-specific $\mathrm{p} K_{\mathrm{a}} \mathrm{s}$, as evidenced by the proposed $\mathrm{K} 125 \mathrm{~L}$ mutant. Moreover, coupling between the protonation and conformational equilibria results in efficient regulation and $\mathrm{pH}$-sensing

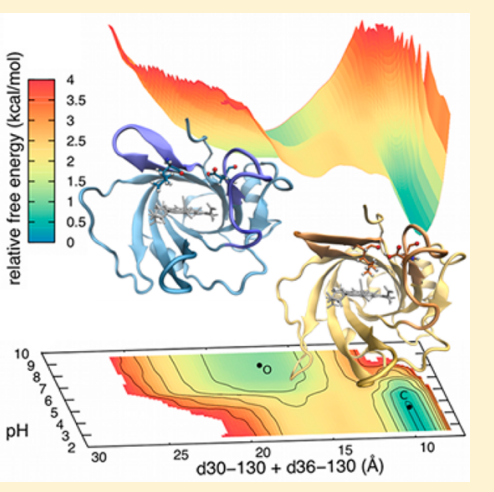
around physiological $\mathrm{pH}$ values only for some combinations of protonation and conformational equilibrium constants, placing constraints on their possible values and leaving a narrow space for protein molecular evolution. The calculations and analysis presented here are of general applicability and provide a guide as to how more complex systems can be studied, offering insight into how $\mathrm{pH}$-regulated allostery works of great value for designing drugs that target $\mathrm{pH}$ sensors and for rational engineering of $\mathrm{pH}$ switches beyond the common histidine trigger.

\section{INTRODUCTION}

$\mathrm{pH}$ is tightly regulated in organisms, even at the subcellular level. ${ }^{1}$ Hence, even small changes in $\mathrm{pH}$ constitute a powerful signaling mechanism that regulates a large number of cellular processes, including proliferation, migration, and viral assembly. ${ }^{2}$ Due to its critical role, $\mathrm{pH}$ dysregulation is associated with diseases like cancer. ${ }^{3} \mathrm{pH}$ differs from other regulation mechanisms such as phosphorylation because its effects are fast, reversible, and do not need to be catalyzed. pH Signaling occurs via $\mathrm{pH}$ sensor proteins, in which changes in $\mathrm{pH}$ can affect key properties such as the specificity of ligand binding, cellular localization, aggregation propensity, and conformation. ${ }^{4}$ Because protein function is intrinsically related to structure, $\mathrm{pH}$-dependent allosteric regulation has important effects on dynamics and catalysis. Understanding how $\mathrm{pH}$-dependent conformational changes take place is key to the development of drugs targeting $\mathrm{pH}$ sensors, ${ }^{3}$ to the success of computational drug discovery strategies, ${ }^{5,6}$ and to the engineering of $\mathrm{pH}$ switches. $3,7-11$

Protein structure, dynamics, and thus, functional $\mathrm{pH}$ dependence arises from the presence of amino acid residues that possess side chains capable of changing their protonation state in response to the environmental $\mathrm{pH}$. These titratable groups are normally present in all proteins, yet not all of them act as $\mathrm{pH}$ sensors. What makes some sites important to $\mathrm{pH}$ regulation while others have a negligible effect? The key factor is that in any protein, only a few amino acids will titrate at the physiological $\mathrm{pH}$ range. As a consequence, in many $\mathrm{pH}$-sensing proteins, histidine residues $\left(\mathrm{p} K_{\mathrm{a}}=6.5\right.$ in water $)$ are critical to $\mathrm{pH}$ regulation ${ }^{2,4,12}$ and histidine switching is a powerful tool to engineer $\mathrm{pH}$-dependent behavior. ${ }^{8-10}$ However, $\mathrm{p} K_{\mathrm{a}} \mathrm{s}$ are strongly influenced by the residue's environment, ${ }^{13}$ which can cause large deviations from typical $\mathrm{p} K_{\mathrm{a}}$ values, ${ }^{14}$ thus expanding nature's resources beyond a single amino acid.

Beyond the need of having at least one site titrating at around physiological (or functional) $\mathrm{pH}$, there are also specific requirements for $\mathrm{pH}$-dependent allosteric regulation arising from the thermodynamic linkage between $\mathrm{pH}$ and protein conformation. ${ }^{15-23}$ Tanford showed that it is necessary to have at least one residue with different conformation-specific $\mathrm{p} K_{\mathrm{a}} \mathrm{s}$, implying that this group must experience significantly different environments in each conformation. ${ }^{24}$ Tuning the environment to achieve the necessary $\mathrm{p} K_{\mathrm{a}}$ shifts around physiological $\mathrm{pH}$ is likely easier in the case of histidine residues, but nature's resourcefulness provides examples of allosteric regulation through other amino acids as well.

Nitrophorin 4 (NP4) is an excellent model for studying $\mathrm{pH}$ regulated allostery because of its relatively small size, the

Received: August 6, 2014

Revised: October 15, 2014

Published: October 15, 2014 
availability of crystal structures at different $\mathrm{pHs},{ }^{25-28}$ and its extensive computational ${ }^{29-33}$ and experimental ${ }^{34-40}$ characterization. This protein is found in the salivary glands of the kissing bug Rhodnius prolixus, which acts as a vector for the parasite causing Chagas' disease (American trypanosomiasis). ${ }^{41}$ The role of NP4 is to selectively release nitric oxide (NO) into the victim's tissues, helping the insect feed by promoting vasodilation. NP4 achieves this selectivity through $\mathrm{pH}$ sensing: at the low $\mathrm{pH}$ of the insect's salivary glands (5.5), NP4 is predominantly in a closed conformation, while at the $\mathrm{pH}$ of the victim's tissues (7.5) it transitions to an open conformation. While the strength of the heme-NO bond is $\mathrm{pH}$-independent, NO release is much more favorable in the open conformation of the protein, ${ }^{30}$ and as a result, the conformational change is the determining factor for $\mathrm{pH}$-dependent regulation.

In the case of NP4, it is well-established that D30 plays a critical role in $\mathrm{pH}$-regulated allosterism. ${ }^{29,32,36}$ In the closed conformation, D30 is buried and forming a hydrogen bond with L130, while in the open conformation, it becomes deprotonated and solvent-exposed, thus losing the interaction. NP4's closed to open transition has an observed $\mathrm{p} K_{\mathrm{a}}$ of $6.5,{ }^{42}$ exactly in the range necessary to achieve regulation around physiological values.

Many computational $\mathrm{p} K_{\mathrm{a}}$ prediction methods are available to identify residues that may be critical for $\mathrm{pH}$ sensing ( $\mathrm{see}^{43}$ for a review), but molecular dynamics (MD)-based methods ${ }^{44-56}$ are ideal for focusing on how the effect of $\mathrm{pH}$ on protonation influences protein conformation and dynamics. In this work, we used a combination of constant $\mathrm{pH} \mathrm{MD}$ (CpHMD) and $\mathrm{pH}$ replica exchange $\mathrm{MD}$ ( $\mathrm{pH}-\mathrm{REMD}) . \mathrm{CpHMD}^{51}$ allows selected residues to explore different protonation states along an $\mathrm{MD}$ trajectory. Protonation changes are attempted regularly and accepted or rejected after evaluating the energetic cost of the proposed change based on the residue's identity, environment, and solvent $\mathrm{pH}$. As a result, the simulation provides an ensemble of properly weighted protonation states and conformations at the $\mathrm{pH}$ of choice. In some cases, achieving a sufficiently converged ensemble using CpHMD is complicated; ${ }^{53,56} \mathrm{pH}$-REMD accelerates convergence and improves conformational state sampling by introducing exchanges between CpHMD simulations running at different $\mathrm{pHs},{ }^{56}$ and the implementation of $\mathrm{MD}$ packages on graphics processing units (GPUs) allows for long simulations at low computational cost. $^{57-61} \mathrm{pH}-\mathrm{REMD}$ allowed us to explore the coupling between conformation and protonation in a large $\mathrm{pH}$ range.

In this work, we will first address the importance of ionizable residues on $\mathrm{pH}$-regulated allostery in $\mathrm{NP} 4$, confirming the key role of $\mathrm{D} 30$. Next, we will describe the $\mathrm{pH}$-dependent freeenergy landscape for the conformational change. This section includes calculations of conformational and acid/base equilibrium constants, as well as estimations of free-energy barriers for the conformational change. The values obtained are discussed in the context of a thermodynamic $\mathrm{pH}$ switch model for optimal $\mathrm{pH}$-sensing. This is followed by calculations that address the possibility of modifying NP4's observed $\mathrm{p} K_{\mathrm{a}}$ without affecting the conformation-specific $p K_{a} s$. Finally, we turn our attention to dynamic aspects of the conformational change, focusing on how the deprotonation of D30 at high $\mathrm{pH}$ drives the conformational change. The type of calculations and analysis presented here are of general applicability and provide a guide as to how more complex systems can be studied. Our results provide valuable insight into how $\mathrm{pH}$-regulated allostery works that can be of great value for designing drugs that target $\mathrm{pH}$ sensors and for rational engineering of $\mathrm{pH}$ switches.

\section{RESULTS AND DISCUSSION}

What is the Effect of NP4 Titratable Residues on the Thermodynamics of the pH-Dependent Conformational Change? The sequence of NP4 contains 18 aspartic acid, 6 glutamic acid, and 3 histidine side chains that may change their protonation state in the $5.5-7.5 \mathrm{pH}$ range where this protein fulfills its biological role. A detailed analysis of the environment for each of these titratable residues in both the open and closed conformations (presented in the Supporting Information) reveals that many of these residues are always solvent exposed or maintain the same environment in both conformations. It is safe to assume that these residues have no effect on the thermodynamics of the conformational change because their $\mathrm{p} K_{\mathrm{a}} \mathrm{s}$ will be the same in both conformations and can be modeled using a fixed protonation state. However, we found that D30, as well as E32, D35, D129, and D132 may have a significant role based on their location in the $\mathrm{AB}$ and $\mathrm{GH}$ loops (Figure 1) and the complex hydrogen bonding interactions

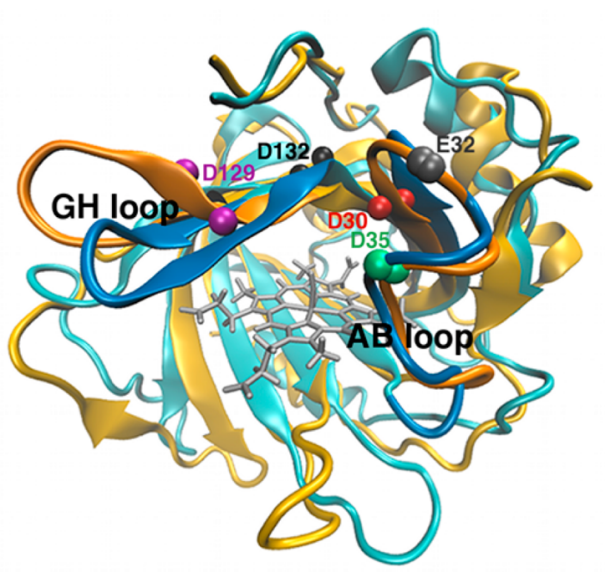

Figure 1. Location of D30, E32, D35, D129, and D132. The AB loop and GH loops are shown in darker colors than the rest of the protein. The closed conformation is depicted in light blue, while the open conformation is shown in yellow.

displayed between them. In order to determine whether any of these residues has significantly different $\mathrm{p} K_{\mathrm{a}} \mathrm{s}$ in the open and closed conformations, we performed 5 independent $\mathrm{CpHMD}$ simulations, each $20 \mathrm{~ns}$ long, of the closed conformation at $\mathrm{pH}$ 5.5 and of the open conformation at $\mathrm{pH} 7.5$, where we titrated these residues. The results, shown in Table 1, reveal that D30 is the only residue that has two significantly different, conformation-dependent $\mathrm{p} K_{\mathrm{a}} \mathrm{s}$. On the basis of these results, all other simulations will consider D30 as the only titratable residue, unless otherwise specified.

Free-Energy Landscape of the pH-Dependent Conformational Change. The recent development of fast $\mathrm{pH}$ REMD methods ${ }^{56}$ makes it possible to study the equilibrium between the different conformations as a function of $\mathrm{pH}$. In previous work, ${ }^{29}$ we identified two key distances that report on whether the hydrogen bond between D30 and L130 is formed $(\mathrm{d} 30-130)$ and the NO-escape route is open $(\mathrm{d} 36-130)$. The different values of these distances in each conformation allow us to use the sum of $\mathrm{d} 30-130$ and $\mathrm{d} 36-130$ as a reaction coordinate that describes salient features of the conformational 
Table 1. Average pKa Values and 95\% Confidence Intervals for D30, E32, D35, D129, and D132 in the Closed and Open Conformations $^{a}$

\begin{tabular}{ccc} 
titratable residue & closed conformation, $\mathrm{pH} 5.5$ & open conformation, $\mathrm{pH} 7.5$ \\
\hline D30 & $8.6 \pm 0.5$ & $5.2 \pm 0.3$ \\
E32 $^{c}$ & $5.5 \pm 0.6$ & $5.4 \pm 0.3$ \\
D35 $^{c}$ & $5.5 \pm 0.3$ & $5.0 \pm 0.2$ \\
D129 $^{c}$ & $3.4 \pm 0.3$ & $3.7 \pm 0.6$ \\
D132 $^{b}$ & $1.9 \pm 1.9$ & $2.6 \pm 0.7$
\end{tabular}

${ }^{a}$ Residue $\mathrm{p} K_{\mathrm{a}} \mathrm{s}$ were estimated based on the fraction of time spent protonated in the corresponding simulation, assuming ergodicity and Henderson-Hasselbach titration curves. ${ }^{b}$ D132 interacts with the backbone amino groups of $\mathrm{T} 3$ and $\mathrm{K} 4$, as well as the hydroxyl group of T3 during most of the dynamics, shifting its $\mathrm{p} K_{\mathrm{a}}$ down substantially. ${ }^{c^{c}}$ The minor upward $\mathrm{p} K_{\mathrm{a}}$ shifts of E32 and D35 are caused by transient interactions with $\mathrm{D} 129$, shifting its $\mathrm{p} K_{\mathrm{a}}$ down as a result.

change. From our simulation data, we can calculate the probability of observing a conformation whose reaction coordinate value lies in a certain range at each $\mathrm{pH}$. Using this probability, we can estimate the relative free energies of each of these structures. Figure 2 shows the resulting $\mathrm{pH}$-dependent

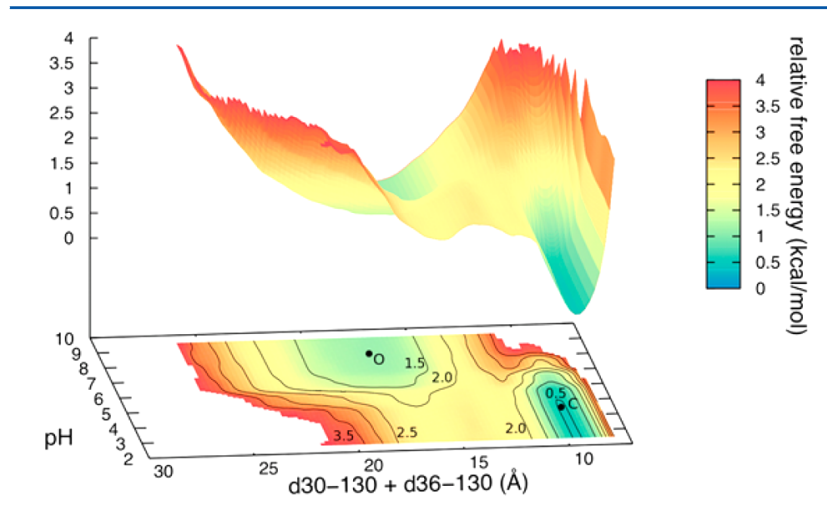

Figure 2. Relative free-energy map for the $\mathrm{pH}$-dependent conformational change. The closed and open basins are labeled $\mathrm{C}$ and $\mathrm{O}$, respectively. Isocontour lines from 0.5 to $3.5 \mathrm{kcal} / \mathrm{mol}$ in $0.5 \mathrm{kcal} / \mathrm{mol}$ increments are shown.

free-energy map. The minimum corresponding to the closed conformation is narrowly distributed around a coordinate value of $9.5 \AA$, while the minimum corresponding to the open structure is much wider, centered at $17 \AA$ and $0.65 \mathrm{kcal} \mathrm{mol}^{-1}$ higher in free energy. Both conformations increase their free energy as we move away from their optimum $\mathrm{pHs}$, but the open conformation remains more energetically accessible than the closed one. At $\mathrm{pH}=6.5$, the free energy of both minima is similar and the transition between them would require an activation free energy of $1.56 \mathrm{kcal} \mathrm{mol}^{-1}$.

The acid/base equilibrium constants can also be determined from the results of the $\mathrm{pH}-\mathrm{REMD}$ simulations. The observed $\mathrm{p} K_{\mathrm{a}}$ can be determined directly from the titration curve obtained by plotting the fraction of deprotonated D30 $\left(f_{\mathrm{D}}\right)$ as a function of $\mathrm{pH}$ (Figure 3), which we fit to the Hill equation:

$$
f_{\mathrm{D}}=\frac{1}{1+10^{n(\mathrm{pKa}-\mathrm{pH})}}
$$

On the basis of the fitting for five different sets of $\mathrm{pH}$-REMD simulations (Figure 3, black titration curve), we found the average observed $\mathrm{p} K_{\mathrm{a}}$ to be $6.2 \pm 0.3$, in excellent agreement with Montfort's experimental value of 6.5 . $^{42}$ Most importantly,

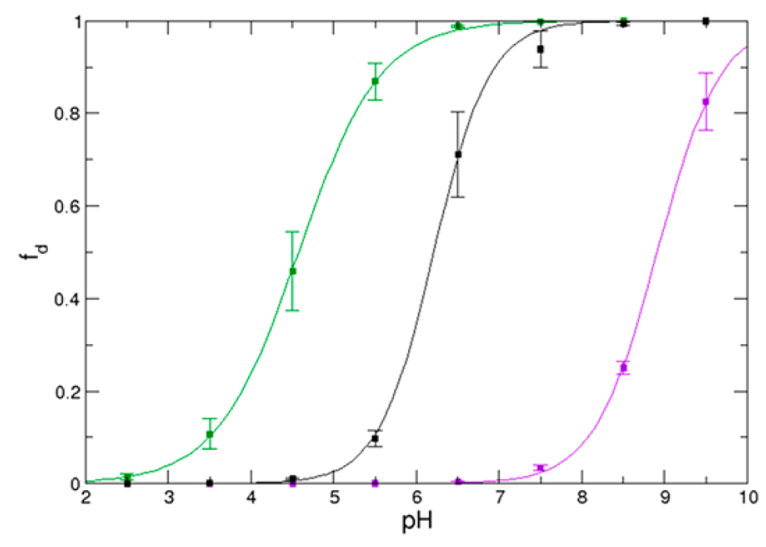

Figure 3. Average titration curves for the closed (purple) and open (green) ensembles and for the complete ensemble (black) obtained using $\mathrm{pH}$-REMD. Each point corresponds to the average $f_{\mathrm{D}}$ at the given $\mathrm{pH}$ computed from the five independent simulations. Error bars correspond to the standard error of the mean.

the observed $\mathrm{p} K_{\mathrm{a}}$ falls in the relevant physiological range necessary for $\mathrm{pH}$ regulation, even though the usual $\mathrm{p} K_{\mathrm{a}}$ of an aspartic acid residue is $\sim 4 .^{14}$

In order to obtain the conformation-specific $\mathrm{p} K_{\mathrm{a}} \mathrm{s}$, we calculated the individual titration curves only for those structures unambiguously assigned as open or closed. At any given time, we determined the conformation of NP4 using a distance criterion based on $\mathrm{d} 30-130$ and $\mathrm{d} 36-130$ (detailed in the Supporting Information). The resulting titration curves were also fit to the Hill equation and are shown in Figure 3. The average $\mathrm{p} K_{\mathrm{a}}$ for the closed conformation is $8.9 \pm 0.01$, while the average $\mathrm{p} K_{\mathrm{a}}$ for the open conformation is $4.5 \pm 0.2$. Both are in good agreement with our previous estimations (8.5 and 4.3, respectively), based on short CpHMD of open and closed structures separately. ${ }^{29}$ As expected based on the environment of $\mathrm{D} 30$, the $\mathrm{p} K_{\mathrm{a}}$ in the closed conformation is considerably up-shifted, while the one for the open conformation is close to the average $\mathrm{p} K_{\mathrm{a}}$ of a solvent-exposed aspartic acid. These calculations confirm that D30 has two different, conformation-specific $\mathrm{p} K_{\mathrm{a}} \mathrm{s}$, as required to regulate pH-dependent allostery. ${ }^{24}$

It is important to note that none of the conformation-specific $\mathrm{p} K_{\mathrm{a}} \mathrm{s}$ correspond to the observed $\mathrm{p} K_{\mathrm{a}}$ of $6.2 \pm 0.3$. In previous work, ${ }^{29}$ we showed that the observed $\mathrm{p} K_{\mathrm{a}}$ is not simply an average of the conformation-specific $\mathrm{p} K_{\mathrm{a}} \mathrm{s}$ because the conformational equilibria, itself $\mathrm{pH}$-dependent, must be taken into account. This can be accomplished using a four-state model, which we summarize in Figure 4 and is applicable to any $\mathrm{pH}$ switch involving two conformations and regulation by a single amino acid. This model has four parameters: the D30 acid/base equilibrium constants in each conformation $\left(K_{\mathrm{a}}\right)$ and the conformational equilibrium constants when D30 is protonated $\left(K_{\mathrm{H}}\right)$ or ionized $\left(K_{\mathrm{D}}\right)$. Of these four, only three are independent and we will show that their values are constrained. Using the equation presented in Figure 4 and the acid/base equilibrium constant values determined from the $\mathrm{pH}$ REMD simulations, we find that the conformational equilibrium constant when D30 is deprotonated is $K_{\mathrm{D}}=580 \pm 400$.

It is also possible to obtain an independent estimation of $K_{D}$ using umbrella sampling to compute the free-energy profile of the conformational change. The nudged elastic band method was used to obtain a minimum energy path connecting the closed and open states at a fixed D30 protonation. This 


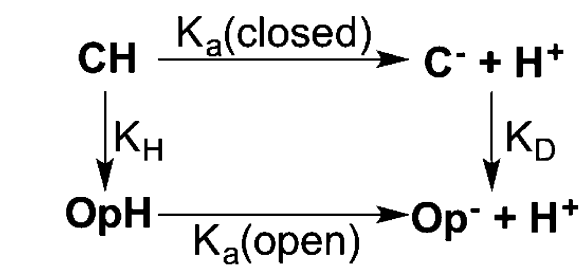

$p K a($ obs $)=p K_{a}$ (open) $+\mathrm{pK}_{\mathrm{a}}($ closed $)-\log \frac{\mathrm{K}_{\mathrm{D}}+1}{\mathrm{~K}_{\mathrm{D}} \mathrm{K}_{\mathrm{a}} \text { (closed) }+\mathrm{K}_{\mathrm{a}} \text { (open) }}$

Figure 4. Thermodynamic model proposed, ${ }^{29}$ and expression for the observed $\mathrm{p} K_{\mathrm{a}}$ in terms of the parameters of the model. $\mathrm{CH}$ : closed state with D30 protonated; $\mathrm{C}^{-}$: closed state with D30 deprotonated; $\mathrm{OpH}$ : open state with $\mathrm{D} 30$ protonated; $\mathrm{Op}^{-}$: open state with $\mathrm{D} 30$ deprotonated. $K_{\mathrm{i}}$ are the equilibrium constants for the reactions in the direction shown by the arrow. $\mathrm{K}_{\mathrm{D}}$ and $\mathrm{K}_{\mathrm{H}}$ are not independent and are related by $K_{\mathrm{H}}=\left[K_{\mathrm{D}} K_{\mathrm{a}}(\right.$ closed $) / K_{\mathrm{a}}($ open $\left.)\right]$.

approach has been used successfully to obtain free-energy barrier estimations in good agreement with experimental data. ${ }^{62}$ The resulting profiles (included in the Supporting Information) allow us to estimate the conformational equilibrium constants based on the free-energy difference between the minima corresponding to the closed and open conformations. The values of $K_{\mathrm{D}}$ obtained are 263 , based on the calculation where D30 is deprotonated, and 83 based on the calculation where $\mathrm{D} 30$ is protonated and using the $\mathrm{p} K_{\mathrm{a}}$ values reported above. These values fall within the range determined from the $\mathrm{pH}$ REMD simulations and support our previous prediction of $K_{\mathrm{D}}$ being of the order of $10^{2}$ to maximize NP4's efficiency. ${ }^{29}$ The free-energy barrier for opening is $5.1 \mathrm{kcal} \mathrm{mol}^{-1}$ in the protonated case and $0.3 \mathrm{kcal} \mathrm{mol}^{-1}$ in the deprotonated case, showing that the opening rate is expected to be $\sim 3000$ faster when D30 deprotonates. In terms of the biological function of $\mathrm{NP} 4$, this difference allows the conformational equilibrium to be established quickly when the protein is injected into the blood, thus allowing fast NO release from the open conformation.

To make a pH-regulated switch work, having different conformation-specific $\mathrm{p} K_{\mathrm{a}} \mathrm{s}$ is crucial but not sufficient. There are also constraints on $K_{\mathrm{D}}$ to achieve proper regulation and thus, it is interesting to assess the effect of $K_{D}$ on the protein's ability to fulfill its biological role: it must be predominantly closed at $\mathrm{pH} 5.5$ to avoid NO release inside the salivary glands of the insect and predominantly open at $\mathrm{pH} 7.5$ to maximize NO release into the victim's tissues. In this context, we can define a score $S$ for the conformational switch in terms of the mole fractions of the open and closed conformation at each $\mathrm{pH}$ (eq 2). In a hypothetical case where NO release is perfectly bimodal, each term would have a value of 1 , resulting in $S_{\max }=$ 2.

$$
S=\left(X_{\text {closed }}-X_{\text {open }}\right)_{\mathrm{pH} 5.5}+\left(X_{\text {open }}-X_{\text {closed }}\right)_{\mathrm{pH} 7.5}
$$

On the basis of the model presented in Figure 4, the score can be written as a function of the two conformation-specific $\mathrm{p} K_{\mathrm{a}} \mathrm{s}$ and $K_{\mathrm{D}}$. For cases like NP4's, where the critical residue for the conformational change is solvent-exposed in one of the conformations, one of the $\mathrm{p} K_{\mathrm{a}} \mathrm{s}$ is fixed and only two other variables remain in our model: the $\mathrm{p} K_{\mathrm{a}}$ in the remaining conformation and $K_{\mathrm{D}}$. Figure 5 shows $S$ as a function of these two variables. The highest $\mathrm{p} K_{\mathrm{a}}$ reported for an aspartic acid residue is $9.2,{ }^{14}$ so we limited the plot to values lower than 9.5 . The plot shows that only certain combinations of $K_{\mathrm{D}}$ and closed $\mathrm{p} K_{\mathrm{a}}$ values result in efficient conformational switching,

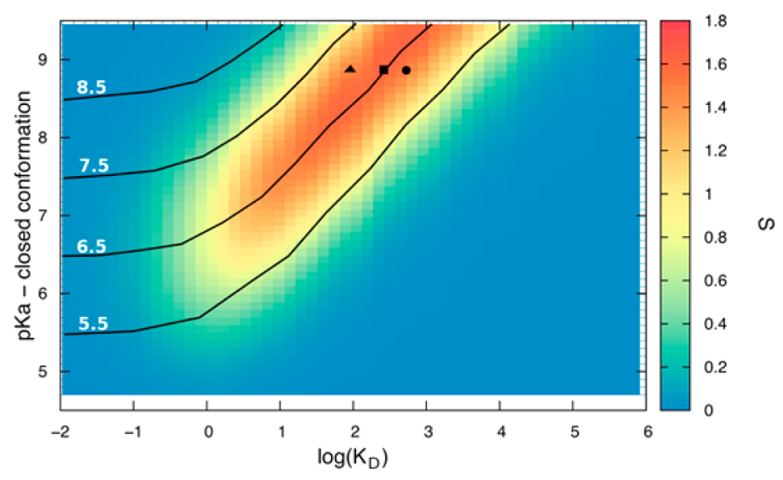

Figure 5. $S$ as a function of the $\mathrm{pK}_{\mathrm{a}}$ of the closed conformation and $K_{\mathrm{D}}$. The calculated values of $K_{\mathrm{D}}$ are shown using a $(\mathrm{pH}-\mathrm{REMD})$, (umbrella sampling, deprotonated case), and $\boldsymbol{\Delta}$ (umbrella sampling, protonated case). Isocontours of observed $\mathrm{p} K_{\mathrm{a}}$ at different values are also shown.

falling in the red region of Figure 5. The $K_{\mathrm{D}}$ values estimated using $\mathrm{pH}$-REMD and umbrella sampling calculations all fall within this range. Outside this region the score drops for different reasons: if $K_{\mathrm{a}}$ (closed) is too high or too low, $\mathrm{pH}$ switching will not happen at $\mathrm{pH} 6.5$; if $K_{\mathrm{D}}$ is too low then $X_{\text {open }}$ at $\mathrm{pH}=7.5$ will be low; and if $K_{\mathrm{D}}$ is too high, this also makes $\mathrm{K}_{\mathrm{H}}$ high, causing a low $X_{\text {closed }}$ at $\mathrm{pH}=5.5$.

This type of analysis could be applied to any other system where only one residue is responsible for $\mathrm{pH}$-dependent regulation, adjusting the score definition to the $\mathrm{pH}$ values relevant for that particular system. If, unlike in this case, one of the conformation-specific $\mathrm{p} K_{\mathrm{a}} \mathrm{s}$ is not known, the analysis can be performed for different combinations of values. The plots obtained have the same general features, but the optimumswitching zone and the iso-p $K_{\mathrm{a}}$ contour lines would shift (see the Supporting Information for an additional example). It is noteworthy that $\mathrm{pH}$-switching is more efficient when the difference between the conformation-specific $\mathrm{p} K_{\mathrm{a}} \mathrm{s}$ is larger, as long as $K_{\mathrm{D}}$ also increases so as to remain in the regulating region of the plot. This implies that even in those cases where histidine residues are responsible for $\mathrm{pH}$-dependent regulation, it is necessary for these sites to have two sufficiently different conformation-specific $\mathrm{p} K_{\mathrm{a}}$ values.

Can We Modify the Observed $\mathrm{p} K_{\mathrm{a}}$ without Affecting the Conformation-Specific $\mathrm{p} K_{\mathrm{a}} s$ ? In accordance with the model we presented, ${ }^{29}$ the observed $\mathrm{p} K_{\mathrm{a}}$ depends on the values of the conformation-specific $\mathrm{p} K_{\mathrm{a}} \mathrm{s}$, and the equilibrium constant $K_{\mathrm{D}}$. This implies that, in principle, it should be possible to modify the observed $\mathrm{p} K_{\mathrm{a}}$ by tweaking only the conformational equilibrium. On the basis of this idea, we devised mutants where the $K_{\mathrm{D}}$ value would be different, but the $\mathrm{p} K_{\mathrm{a}} \mathrm{s}$ of D30 would remain the same. V36 is an obvious target for mutations, since we know that the packing of its side-chain against that of L130 stabilizes the closed conformation. However, the V36 mutants attempted either had no significant effect on the observed $\mathrm{pK}_{\mathrm{a}}$ (e.g., V36A) or affected both the observed $\mathrm{p} K_{\mathrm{a}}$ and the conformation-specific $\mathrm{p} K_{\mathrm{a}} \mathrm{s}$ due to changes in the solvent exposure of D30 (e.g., V36G). Because of this, we turned our attention to $\mathrm{K} 125$, a residue located in the GH loop, away from D30. This residue forms a hydrogen bond with the heme $50 \%$ of the time in the closed conformation, and the hydrocarbon part of its side chain is packed against L130. In the open conformation, it forms this hydrogen bond only $10 \%$ of the time, and it is more solvent-exposed. In order to validate 
that a possible protonation change in K125 would not affect the results, we performed a $\mathrm{pH}$-REMD simulation where both K125 and D30 were allowed to titrate and observed no differences with respect to the simulations where only D30 was titratable (details available in the Supporting Information). In the K125L mutant, the closed conformation is still stabilized by packing of L125 against L130, but the hydrophobic nature of the leucine side chain relatively destabilizes the open conformation, in which it becomes solvent-exposed. Figure 6

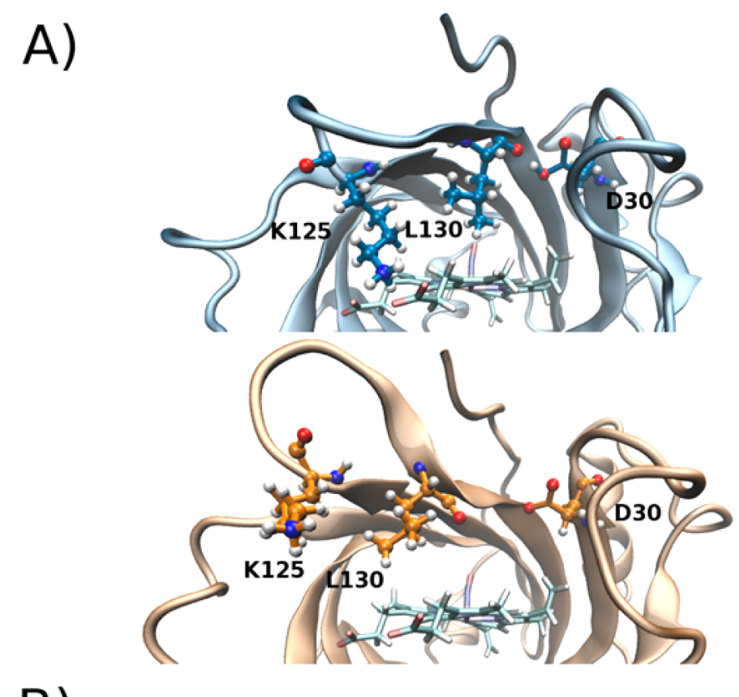

B)

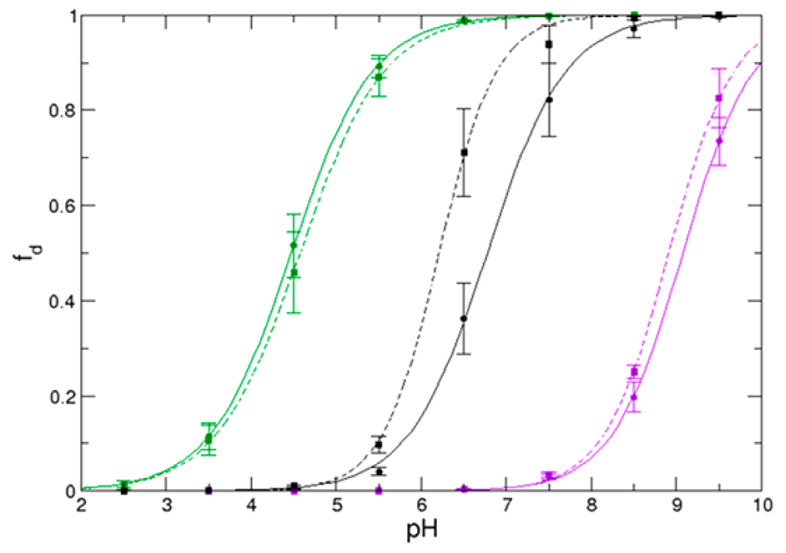

Figure 6. (A) Location of K125 in the closed (blue) and the open (orange) conformations. L130, D30, and the heme are also shown. (B) Titration curves of K125L ( lines). The values shown are the averages of five different independent $\mathrm{pH}$-REMD simulations; error bars correspond to the standard error of the mean. Green: open ensemble; purple: closed ensemble; black: complete ensemble.

shows that the titration curve obtained for the K125L mutant differs significantly from the WT curve at $\mathrm{pH}$ 6.5. As a result, the observed $\mathrm{p} K_{\mathrm{a}}$ for $\mathrm{K} 125 \mathrm{~L}$ is $6.8 \pm 0.4$, while the $\mathrm{p} K_{\mathrm{a}}$ of $\mathrm{D} 30$ in the closed conformation is $8.9 \pm 0.01$ and in the open conformation is $4.5 \pm 0.2$. The $K_{\mathrm{D}}$ calculated from these values is $250 \pm 160$. Although this value is not statistically different from the one observed for the WT due to the large uncertainty in its estimation, it is the only parameter that is changing enough to explain the changes in the titration curve. Taken together, our results confirm that it is possible to modulate the observed $\mathrm{p} K_{\mathrm{a}}$ by modifying $K_{\mathrm{D}}$ while keeping the conformationspecific $\mathrm{p} K_{\mathrm{a}} \mathrm{s}$ constant.

Dynamics of the pH-Dependent Conformational Change. In order to study the details of how the conformational change from closed to open takes place, we performed eight $40 \mathrm{~ns}$ simulations where the closed conformation is initially placed at a $\mathrm{pH}$ of 7.5 , at which the open conformation is more stable. For those simulations where the conformational change took place, we mapped the D30 protonated fraction with respect to $\mathrm{d} 30-130$, which reports on whether the key hydrogen bond is formed and $\mathrm{d} 36-130$, which increases when the protein opens to facilitate NO escape, as the simulation progressed (Figure 7). Our results show that deprotonation

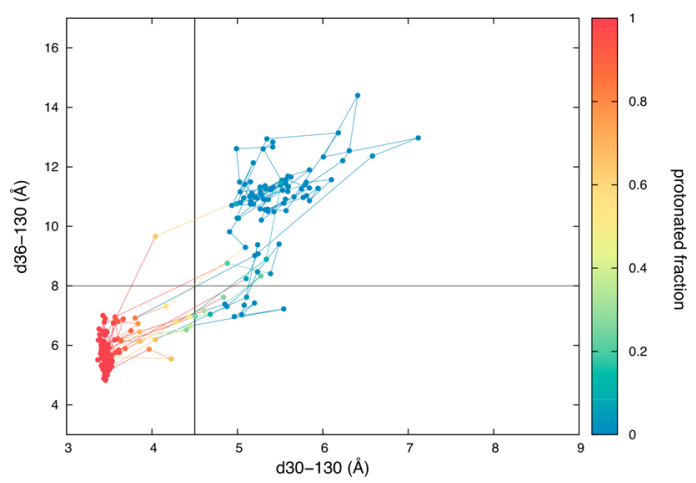

Figure 7. Mapping of the protonated fraction as a function of $\mathrm{d} 30-$ 130 and $\mathrm{d} 36-130$ for one of the trajectories at $\mathrm{pH} 7.5$ that starts from the closed conformation. The plots for all trajectories are available in the Supporting Information. Each data point represents the average value for $0.2 \mathrm{~ns}$.

begins while the protein can still be considered closed (yellow and orange points in the left lower quadrant), after a slight increase in the hydrogen-bonding distance between D30 and L130 that can be interpreted as the opening necessary for a water molecule to be able to interact with D30 and change its protonation. In the majority of cases, the transition to the open conformation takes place through the lower right quadrant. This is consistent with a conformational change driven by a change in protonation of $\mathrm{D} 30$, since $\mathrm{d} 30-130$ increases first, and $\mathrm{d} 36-130$ increases later as a consequence, opening the protein. Once the system crosses over to the open quadrant, it explores a larger range of conformations, consistent with the fact that the open minimum is much wider than the closed one and the number of possible open structures is expected to be much larger than the number of possible closed configurations. In other words, NP4's allosteric transition occurs in two steps: first the D30 $\mathrm{pH}$ switch is locally opened, fixing D30 in the charged, solvent-exposed configuration and then the $A B$ and $\mathrm{GH}$ loops open, paving the way for $\mathrm{NO}$ escape. It is important to note that because of how the protonation changes are treated in our simulations, we are working under the assumption that protonation changes are much faster than conformational changes. This assumption is normally employed when studying the kinetics of similar systems. ${ }^{63}$

One interesting possibility is that while E32, D35, D129, and D132 play no role on $\mathrm{pH}$-regulated allostery, they may play a role in the details of how the conformational change takes place. In order to consider this, we performed the same type of simulations but now allowing all these residues to change protonation. Out of the five simulations where the conforma- 
tional change took place, in three of them, there are no noticeable differences with respect to the case where only D30 is allowed to titrate. In the remaining two, we observed that the hydrogen bond between E32 and D129 was formed most of the time before $\mathrm{D} 30$ became deprotonated, causing an increase in the d30-130 distance. However, because the E32-D129 bond was formed, d36-130 did not reach values typical of the open conformation until this bond was broken (see the Supporting Information for more details). These results show that although we determined that E32, D35, D129, and D132 have no effect on the thermodynamics of the conformational change, they might have an effect on its kinetics, which we are not able to quantify based solely on these simulations.

\section{CONCLUSIONS}

In this work, we present a comprehensive study of the $\mathrm{pH}$ regulated allosteric change that controls NP4 activity and allows it to fulfill its function. Our results show that D30 is the only amino acid whose protonation equilibrium is thermodynamically linked to the conformational change, since it is the only residue that has two significantly different conformation-specific $\mathrm{p} K_{\mathrm{a}} \mathrm{s}$. In accordance with our calculations, the $\mathrm{p} K_{\mathrm{a}} \mathrm{s}$ for the closed and open conformations are $8.9 \pm 0.01$ and $4.5 \pm 0.2$, respectively, confirming that D30 has two significantly different conformation-specific $\mathrm{p} K_{\mathrm{a}} \mathrm{s}$ due to the different environments it experiences in each state. This property of D30 is what determines its crucial role in regulating $\mathrm{pH}$-dependent allostery.

Our simulations allowed us to explore the $\mathrm{pH}$-dependent free-energy landscape of NP4's conformational change. While the minimum for the closed conformation is narrow, the one for the open case is wider and shallower. At $\mathrm{pH} 6.5$, both states have similar free energies and the barrier for the transition between them is low. We also computed the observed $\mathrm{p} K_{\mathrm{a}}$ to be $6.2 \pm 0.3$, in excellent agreement with experimental results ${ }^{42}$ and within the range necessary for regulation around physiological $\mathrm{pH}$. Our estimations of $K_{\mathrm{D}}$ using different types of calculations all yield values on the order of $10^{2}$, which fall in the range required for efficient $\mathrm{pH}$ switching. Our model for how the observed $\mathrm{p} K_{\mathrm{a}}$ arises from the coupling between conformational and protonation equilibria suggests the interesting possibility of being able to tune this value without changing the conformation-specific $\mathrm{p} K_{\mathrm{a}} \mathrm{s}$. Our calculations confirm this for the K125L mutant.

In accordance with our data, NP4's allosteric transition occurs in two steps: first the $\mathrm{D} 30 \mathrm{pH}$ switch is locally opened, allowing D30 to deprotonate, and then the $\mathrm{AB}$ and $\mathrm{GH}$ loops open, paving the way for NO escape. This is consistent with D30 acting as the allosteric trigger because when it becomes charged at $\mathrm{pH} 7.5$, breaking the hydrogen bond to L130, this results in an unfavorable electrostatic interaction that is the driving force for the conformational change. Our results suggest that E32 and D129 may play a role in the kinetics of this event but do not affect the equilibrium distributions.

On the basis of the results obtained for NP4, we present a general thermodynamic model of a protein $\mathrm{pH}$ switch between two conformations regulated by a single amino acid. The fourstate model, based on a single titratable residue and two protein conformations, allowed us to analyze the requirements for efficient conformational switching. Our data shows that the switch works only for certain combinations of values for the acid/base and conformational equilibrium constants, which maximize the populations of each conformation at the two $\mathrm{pH}$ values of relevance. This analysis can be applied to any other case where the conformational change is driven by a single amino acid that is solvent exposed in one of the conformations. ${ }^{64}$ It is worth noting that even in cases where a histidine residue is responsible for $\mathrm{pH}$-dependent regulation, having two sufficiently different conformation-specific $\mathrm{p} K_{\mathrm{a}} \mathrm{s}$ is a requirement for efficient conformational switching.

Our results show clearly that efficient $\mathrm{pH}$-regulated allostery can be accomplished for residues other than histidine, a fact that seems to be given little attention when considering how to engineer $\mathrm{pH}$ switches, which are largely histidine-based ${ }^{8-10}$ or when analyzing what residues play important roles in $\mathrm{pH}$ sensors that could be interesting drug targets. Garcia-Moreno and co-workers have laid the foundations for tuning the $\mathrm{p} K_{\mathrm{a}}$ of buried ionizable groups, ${ }^{7}$ making it possible in principle to design systems where $\mathrm{pH}$-regulated allostery works in a similar way as in NP4: having a titratable residue that is solventexposed in one conformation and tuning the $\mathrm{p} K_{\mathrm{a}}$ in the state where it is buried to achieve an observed $\mathrm{p} K_{\mathrm{a}}$ in any desired range between them.

\section{METHODS}

General Considerations. All simulations were performed using the AMBER12 suite. ${ }^{65}$ The Amber ff99SB force field ${ }^{66}$ parameters were used for all residues except the heme, for which we employed parameters developed and tested by our group. ${ }^{67}$ The SHAKE algorithm was used to keep bonds involving $\mathrm{H}$ atoms at their equilibrium length. ${ }^{68}$ Newton's equations were integrated with a 2 fs time step, and frames were collected at 1 ps intervals. All simulations used different random seeds to avoid synchronization artifacts. ${ }^{69}$ Initial structures for each conformation were derived from $20 \mathrm{~ns}$ long dynamics with fixed protonation states, which we describe in detail elsewhere. ${ }^{29}$ These started from PDB $=1 \mathrm{X} 8 \mathrm{O}(1.01 \AA)$ for the closed conformation, and PDB $=1$ X8N (1.08 $\AA$ ) for the open conformation. ${ }^{25}$

Constant pH Molecular Dynamics (CpHMD). Starting topologies were built using the tleap module of AmberTools 13, and the initial protonation of titratable residues was chosen in agreement with previous work. ${ }^{25,29-31}$ The initial structure was minimized for 1000 steps using $10 \mathrm{kcal} \mathrm{mol}^{-1} \AA^{-2}$ restraints on the backbone, and then heated using a linear gradient from 10 to $300 \mathrm{~K}$ during $0.2 \mathrm{~ns}$ and maintained at the final temperature during $0.2 \mathrm{~ns}$ using the same backbone restraints. Finally, we performed $1 \mathrm{~ns}$ of dynamics with $1 \mathrm{kcal} \mathrm{mol}^{-1} \AA^{-2}$ restraints on the backbone. All production simulations were performed using the pmemd.cuda module of AMBER $12 .{ }^{65}$ We used the Generalized Born implicit solvent model, $^{70}$ a $0.1 \mathrm{M}$ salt concentration, an infinite cutoff for nonbonded interactions, and a $300 \mathrm{~K}$ temperature maintained using Langevin dynamics with a collision frequency of $10 \mathrm{ps}^{-1}$. Using the CpHMD method, ${ }^{51}$ we attempted a change in protonation state every 5 steps. Amino acid $\mathrm{p} K_{\mathrm{a}} \mathrm{s}$ were estimated based on the fraction of time spent protonated, assuming ergodicity and HendersonHasselbach titration curves $\left(\mathrm{p} K_{\mathrm{a}}=\mathrm{pH}-\log \left(\left[\mathrm{X}^{-}\right] /[\mathrm{HX}]\right)\right)$.

pH-Replica Exchange Molecular Dynamics (pHREMD). Starting topologies were built using the tleap module of AmberTools 13, and the initial protonation of titratable residues was chosen in agreement with previous work. ${ }^{25,30,31}$ All $\mathrm{pH}$-REMD simulations were run using 8 equally spaced replicas in the $2.5-9.5 \mathrm{pH}$ range. The initial closed and open structures were minimized and equilibrated like those for CpHMD. The $\mathrm{pH}-\mathrm{REMD}$ simulations were started using the equilibrated closed structure as the initial structure for replicas in the 2.5- 
$5.5 \mathrm{pH}$ range, while the remaining replicas started from the equilibrated open structure. Each replica was initially simulated at its corresponding $\mathrm{pH}$ for $1 \mathrm{~ns}$, using the pmemd.cuda.MPI module, before any exchange between replicas was allowed. During the $50 \mathrm{~ns}$ production stage, we attempted to exchange replicas every 1000 steps. All other simulation parameters were the same as for CpHMD.

Nudged Elastic Band and Umbrella Sampling Simulations. Starting structures were obtained from $20 \mathrm{~ns}$ long molecular dynamics simulations in implicit solvent ${ }^{70}$ at $300 \mathrm{~K}$ of the open and closed conformation with D30 either constantly protonated or deprotonated. For those simulations where the structure would not be stable ${ }^{32}$ (closed with D30 deprotonated, open with D30 protonated), we used 1 kcal $\mathrm{mol}^{-1} \AA^{-2}$ restraints on the backbone to prevent the conformational change. The NEB method was used to obtain structures within a path that links the closed and open conformations for each protonation state. ${ }^{62}$ Path calculations for each protonation state were performed by linking 12 copies of the closed structure and 12 copies of the open structure. NEB forces were calculated on all atoms, and a Langevin thermostat was used. The 6 stage simulated annealing path optimization procedure employed is similar to the one used by Bergonzo et al.: ${ }^{62}$ (1) 80 ps equilibration at $300 \mathrm{~K}$ with $10 \mathrm{kcal}$ $\mathrm{mol}^{-1} \AA^{-2}$ spring forces and a $100 \mathrm{ps}^{-1}$ collision frequency to ensure rapid uniform heating and a viscosity that stabilizes the system against the force of the springs; (2) 500 ps equilibration at the same temperature, increasing spring forces to $50 \mathrm{kcal}$ $\mathrm{mol}^{-1} \AA^{-2}$ and decreasing the collision frequency to $75 \mathrm{ps}^{-1}$; (3) stepwise heating from 300 to $350 \mathrm{~K}$ during $80 \mathrm{ps}$, with a spring constant of $25 \mathrm{kcal} \mathrm{mol}^{-1} \AA^{-2}$ and the same collision frequency as stage 2 ; (4) 500 ps equilibration at $350 \mathrm{~K}$; (5) stepwise cooling to $300 \mathrm{~K}$ over $100 \mathrm{ps}$; and (6) final equilibration at $300 \mathrm{~K}$ for $1000 \mathrm{ps}$, using the same collision frequency and spring constant as in stage 3 . This calculation allowed us to identify the distance between CB in V36 and CG in L130 (d36-130) as a suitable driving coordinate for subsequent umbrella sampling given its linear variation (see the Supporting Information).

Each structure obtained from NEB was solvated using TIP3P waters, and the resulting water boxes were relaxed by subjecting them to 2000 steps of minimization, followed by 100 ps of linear heating from 50 to $300 \mathrm{~K}$ and $350 \mathrm{ps}$ of $N, V, T$ dynamics at $300 \mathrm{~K}$, all using large $100 \mathrm{kcal} \mathrm{mol}^{-1} \AA^{-2}$ restraints on the protein. Umbrella sampling was performed using $10 \mathrm{kcal} \mathrm{mol}^{-1}$ $\AA^{-2}$ restraints on the $\mathrm{d} 36-130$. Each umbrella was equilibrated for 100 ps, followed by $2.5 \mathrm{~ns}$ of production simulations. WHAM analysis was used to calculate free energies. ${ }^{71}$ All explicit solvent simulations were performed using periodic boundary conditions and Langevin dynamics with a $2 \mathrm{ps}^{-1}$ collision frequency.

\section{ASSOCIATED CONTENT}

\section{S Supporting Information}

Analysis of environment of titratable residues, free-energy profiles at $\mathrm{pH} 5.5$ and 7.5 from $\mathrm{pH}$-REMD simulations, distance criterion for conformational sorting, additional detail for nudged elastic band and umbrella sampling simulations, additional information for $\mathrm{pH}$-REMD simulations on NP4 mutants, effect of additional titratable residues on the dynamics of the conformational change, additional example of conformational switching score calculations, and convergence informa- tion on the $\mathrm{pH}$-REMD simulations. This material is available free of charge via the Internet at http://pubs.acs.org.

\section{AUTHOR INFORMATION}

\section{Corresponding Author}

*E-mail: roitberg@ufl.edu.

\section{Notes}

The authors declare no competing financial interest.

\section{ACKNOWLEDGMENTS}

Computational support was provided by NCSA BlueWaters (NSF OCI 07-25070 and PRAC OCI-1036208) and the University of Florida High Performance Computing Center. The authors acknowledge support from NSF OCI-1147910, Grant "Raices Siembra 2014/15" from MINCyT Argentina, PICT-2010-416, and PIP 2012-112 20110100850 to M.A.M. N.V.D.R is an HHMI International Student Research Fellow. M.A.M. is a member of CONICET.

\section{REFERENCES}

(1) Garcia-Moreno, B. Adaptations of Proteins to Cellular and Subcellular pH. J. Biol. 2009, 8, 98.

(2) Schönichen, A.; Webb, B. A.; Jacobson, M. P.; Barber, D. L. Considering Protonation as a Posttranslational Modification Regulating Protein Structure and Function. Annu. Rev. Biophys. 2013, 42, 289-314.

(3) Webb, B. A.; Chimenti, M.; Jacobson, M. P.; Barber, D. L. Dysregulated pH: A Perfect Storm for Cancer Progression. Nat. Rev. Cancer 2011, 11, 671-677.

(4) Srivastava, J.; Barber, D. L.; Jacobson, M. P. Intracellular $\mathrm{pH}$ Sensors: Design Principles and Functional Significance. Physiology 2007, 22, 30-39.

(5) Polgár, T.; Keserü, G. M. Virtual Screening for B-Secretase (BACE1) Inhibitors Reveals the Importance of Protonation States at Asp32 and Asp228. J. Med. Chem. 2005, 48, 3749-3755.

(6) Polgár, T.; Magyar, C.; Simon, I.; Keserü, G. M. Impact of Ligand Protonation on Virtual Screening against B-Secretase (BACE1). J. Chem. Inf. Model. 2007, 47, 2366-2373.

(7) Pey, A. L.; Rodriguez-Larrea, D.; Gavira, J. A.; Garcia-Moreno, B.; Sanchez-Ruiz, J. M. Modulation of Buried Ionizable Groups in Proteins with Engineered Surface Charge. J. Am. Chem. Soc. 2010, 132, $1218-1219$.

(8) Zimenkov, Y.; Dublin, S. N.; Ni, R.; Tu, R. S.; Breedveld, V.; Apkarian, R. P.; Conticello, V. P. Rational Design of a Reversible pHResponsive Switch for Peptide Self-Assembly. J. Am. Chem. Soc. 2006, 128, 6770-6771.

(9) Sarkar, C. A.; Lowenhaupt, K.; Horan, T.; Boone, T. C.; Tidor, B.; Lauffenburger, D. A. Rational Cytokine Design for Increased Lifetime and Enhanced Potency Using $\mathrm{pH}$-Activated "Histidine Switching". Nat. Biotechnol. 2002, 20, 908-913.

(10) Murtaugh, M. L.; Fanning, S. W.; Sharma, T. M.; Terry, A. M.; Horn, J. R. A Combinatorial Histidine Scanning Library Approach to Engineer Highly pH-Dependent Protein Switches. Protein Sci. 2011, $20,1619-1631$.

(11) Idili, A.; Vallée-Bélisle, A.; Ricci, F. Programmable pH-Triggered DNA Nanoswitches. J. Am. Chem. Soc. 2014, 136, 5836-5839.

(12) Srivastava, J.; Barreiro, G.; Groscurth, S.; Gingras, A. R.; Goult, B. T.; Critchley, D. R.; Kelly, M. J. S.; Jacobson, M. P.; Barber, D. L. Structural Model and Functional Significance of $\mathrm{pH}$-Dependent Talin-Actin Binding for Focal Adhesion Remodeling. Proc. Natl. Acad. Sci. U.S.A. 2008, 105, 14436-14441.

(13) Harris, T. K.; Turner, G. J. Structural Basis of Perturbed pKa Values of Catalytic Groups in Enzyme Active Sites. IUBMB Life 2002, 53, 85-98.

(14) Grimsley, G. R.; Scholtz, J. M.; Pace, C. N. A Summary of the Measured $\mathrm{pK}$ Values of the Ionizable Groups in Folded Proteins. Protein Sci. 2009, 18, 247-251. 
(15) Wyman J., Jr. Heme Proteins. Advances in Protein Chemistry; Anson, M. L., Edsall, J. T., Eds.; Academic Press: Waltham, MA, 1948; pp 407-531.

(16) Yang, A.-S.; Honig, B. On the pH Dependence of Protein Stability. J. Mol. Biol. 1993, 231, 459-474.

(17) Antosiewicz, J.; McCammon, J. A.; Gilson, M. K. Prediction of pH-Dependent Properties of Proteins. J. Mol. Biol. 1994, 238, 415436.

(18) Bashford, D.; Karplus, M. Multiple-Site Titration Curves of Proteins: An Analysis of Exact and Approximate Methods for Their Calculation. J. Phys. Chem. 1991, 95, 9556-9561.

(19) Georgescu, R. E.; Alexov, E. G.; Gunner, M. R. Combining Conformational Flexibility and Continuum Electrostatics for Calculating $\mathrm{pK}_{\mathrm{a}} \mathrm{s}$ in Proteins. Biophys. J. 2002, 83, 1731-1748.

(20) Karp, D. A.; Gittis, A. G.; Stahley, M. R.; Fitch, C. A.; Stites, W. E.; García-Moreno, E. B. High Apparent Dielectric Constant Inside a Protein Reflects Structural Reorganization Coupled to the Ionization of an Internal Asp. Biophys. J. 2007, 92, 2041-2053.

(21) Whitten, S. T.; García-Moreno, E. B.; Hilser, V. J. Local Conformational Fluctuations Can Modulate the Coupling between Proton Binding and Global Structural Transitions in Proteins. Proc. Natl. Acad. Sci. U.S.A. 2005, 102, 4282-4287.

(22) Shi, C.; Wallace, J. A.; Shen, J. K. Thermodynamic Coupling of Protonation and Conformational Equilibria in Proteins: Theory and Simulation. Biophys. J. 2012, 102, 1590-1597.

(23) Goh, G. B.; Laricheva, E. N.; Brooks, C. L. Uncovering pHDependent Transient States of Proteins with Buried Ionizable Residues. J. Am. Chem. Soc. 2014, 136, 8496-8499.

(24) Tanford, C. Ionization-Linked Changes in Protein Conformation. I. Theory. J. Am. Chem. Soc. 1961, 83, 1628-1634.

(25) Kondrashov, D. A.; Roberts, S. A.; Weichsel, A.; Montfort, W. R. Protein Functional Cycle Viewed at Atomic Resolution: Conformational Change and Mobility in Nitrophorin 4 as a Function of $\mathrm{pH}$ and NO Binding. Biochemistry 2004, 43, 13637-13647.

(26) Weichsel, A.; Andersen, J. F.; Champagne, D. E.; Walker, F. A.; Montfort, W. R. Crystal Structures of a Nitric Oxide Transport Protein from a Blood-Sucking Insect. Nat. Struct. Mol. Biol. 1998, 5, 304-309.

(27) Weichsel, A.; Andersen, J. F.; Roberts, S. A.; Montfort, W. R. Nitric Oxide Binding to Nitrophorin 4 Induces Complete Distal Pocket Burial. Nat. Struct. Mol. Biol. 2000, 7, 551-554.

(28) Maes, E. M.; Roberts, S. A.; Weichsel, A.; Montfort, W. R. Ultrahigh Resolution Structures of Nitrophorin 4: Heme Distortion in Ferrous CO and NO Complexes. Biochemistry 2005, 44, 1269012699.

(29) Di Russo, N. V.; Estrin, D. A.; Martí, M. A.; Roitberg, A. E. pHDependent Conformational Changes in Proteins and Their Effect on Experimental $\mathrm{p} K_{\mathrm{a}} \mathrm{s}$ : The Case of Nitrophorin 4. PLoS Comput. Biol. 2012, 8, e1002761.

(30) Martí, M. A.; Lebrero, M. C. G.; Roitberg, A. E.; Estrin, D. A. Bond or Cage Effect: How Nitrophorins Transport and Release Nitric Oxide. J. Am. Chem. Soc. 2008, 130, 1611-1618.

(31) Menyhárd, D. K.; Keserü, G. M. Protonation State of Asp30 Exerts Crucial Influence over Surface Loop Rearrangements Responsible for NO Release in Nitrophorin 4. FEBS Lett. 2005, 579, 5392-5398.

(32) Martí, M. A.; Estrin, D. A.; Roitberg, A. E. Molecular Basis for the $\mathrm{pH}$ Dependent Structural Transition of Nitrophorin 4. J. Phys. Chem. B 2009, 113, 2135-2142.

(33) Swails, J. M.; Meng, Y.; Walker, F. A.; Marti, M. A.; Estrin, D. A.; Roitberg, A. E. pH-Dependent Mechanism of Nitric Oxide Release in Nitrophorins 2 and 4. J. Phys. Chem. B 2009, 113, 1192-1201.

(34) Montfort, W. R.; Weichsel, A.; Andersen, J. F. Nitrophorins and Related Antihemostatic Lipocalins from Rhodnius Prolixus and Other Blood-Sucking Arthropods. Biochim. Biophys. Acta, Protein Struct. Mol. Enzymol. 2000, 1482, 110-118.

(35) Champagne, D. E.; Nussenzveig, R. H.; Ribeiro, J. M. C. Purification, Partial Characterization, and Cloning of Nitric OxideCarrying Heme Proteins (Nitrophorins) from Salivary Glands of the
Blood-Sucking Insect Rhodnius Prolixus. J. Biol. Chem. 1995, 270, 8691-8695.

(36) Maes, E. M.; Weichsel, A.; Andersen, J. F.; Shepley, D.; Montfort, W. R. Role of Binding Site Loops in Controlling Nitric Oxide Release: Structure and Kinetics of Mutant Forms of Nitrophorin 4. Biochemistry 2004, 43, 6679-6690.

(37) Nienhaus, K.; Maes, E. M.; Weichsel, A.; Montfort, W. R.; Nienhaus, G. U. Structural Dynamics Controls Nitric Oxide Affinity in Nitrophorin 4. J. Biol. Chem. 2004, 279, 39401-39407.

(38) Abbruzzetti, S.; He, C.; Ogata, H.; Bruno, S.; Viappiani, C.; Knipp, M. Heterogeneous Kinetics of the Carbon Monoxide Association and Dissociation Reaction to Nitrophorin 4 and 7 Coincide with Structural Heterogeneity of the Gate-Loop. J. Am. Chem. Soc. 2012, 134, 9986-9998.

(39) Benabbas, A.; Ye, X.; Kubo, M.; Zhang, Z.; Maes, E. M.; Montfort, W. R.; Champion, P. M. Ultrafast Dynamics of Diatomic Ligand Binding to Nitrophorin 4. J. Am. Chem. Soc. 2010, 132, 28112820.

(40) Cheng, M.; Brookes, J. F.; Montfort, W. R.; Khalil, M. pHDependent Picosecond Structural Dynamics in the Distal Pocket of Nitrophorin 4 Investigated by 2D IR Spectroscopy. J. Phys. Chem. B 2013, 117, 15804-15811.

(41) Kirchhoff, L. V. American Trypanosomiasis (Chagas' Disease): A Tropical Disease Now in the United States. N. Engl. J. Med. 1993, $329,639-644$.

(42) Andersen, J. F.; Ding, X. D.; Balfour, C.; Shokhireva, T. K.; Champagne, D. E.; Walker, F. A.; Montfort, W. R. Kinetics and Equilibria in Ligand Binding by Nitrophorins 1-4: Evidence for Stabilization of a Nitric Oxide-Ferriheme Complex through a LigandInduced Conformational Trap. Biochemistry 2000, 39, 10118-10131.

(43) Alexov, E.; Mehler, E. L.; Baker, N. M.; Baptista, A.; Huang, Y.; Milletti, F.; Erik Nielsen, J.; Farrell, D.; Carstensen, T.; Olsson, M. H. M.; et al. Progress in the Prediction of pKa Values in Proteins. Proteins: Struct., Funct., Bioinf. 2011, 79, 3260-3275.

(44) Van Vlijmen, H. W. T.; Schaefer, M.; Karplus, M. Improving the Accuracy of Protein $\mathrm{pKa}$ Calculations: Conformational Averaging versus the Average Structure. Proteins: Struct., Funct., Bioinf. 1998, 33, 145-158.

(45) Kuhn, B.; Kollman, P. A.; Stahl, M. Prediction of pKa Shifts in Proteins Using a Combination of Molecular Mechanical and Continuum Solvent Calculations. J. Comput. Chem. 2004, 25, 18651872.

(46) Kato, M.; Warshel, A. Using a Charging Coordinate in Studies of Ionization Induced Partial Unfolding. J. Phys. Chem. B 2006, 110, $11566-11570$.

(47) Khandogin, J.; Brooks, C. L. Toward the Accurate FirstPrinciples Prediction of Ionization Equilibria in Proteins. Biochemistry 2006, 45, 9363-9373.

(48) Lee, M. S.; Salsbury, F. R.; Brooks, C. L. Constant-pH Molecular Dynamics Using Continuous Titration Coordinates. Proteins: Struct., Funct., Bioinf. 2004, 56, 738-752.

(49) Khandogin, J.; Brooks, C. L. Constant pH Molecular Dynamics with Proton Tautomerism. Biophys. J. 2005, 89, 141-157.

(50) Wallace, J. A.; Shen, J. K. Continuous Constant pH Molecular Dynamics in Explicit Solvent with pH-Based Replica Exchange. J. Chem. Theory Comput. 2011, 7, 2617-2629.

(51) Mongan, J.; Case, D. A.; McCammon, J. A. Constant pH Molecular Dynamics in Generalized Born Implicit Solvent. J. Comput. Chem. 2004, 25, 2038-2048.

(52) De Oliveira, C. A. F.; Hamelberg, D.; McCammon, J. A. Coupling Accelerated Molecular Dynamics Methods with Thermodynamic Integration Simulations. J. Chem. Theory Comput. 2008, 4, $1516-1525$

(53) Williams, S. L.; de Oliveira, C. A. F.; McCammon, J. A. Coupling Constant $\mathrm{pH}$ Molecular Dynamics with Accelerated Molecular Dynamics. J. Chem. Theory Comput. 2010, 6, 560-568.

(54) Baptista, A. M.; Martel, P. J.; Petersen, S. B. Simulation of Protein Conformational Freedom as a Function of $\mathrm{pH}$ : Constant-pH 
Molecular Dynamics Using Implicit Titration. Proteins: Struct., Funct., Bioinf. 1997, 27, 523-544.

(55) Baptista, A. M.; Teixeira, V. H.; Soares, C. M. Constant-pH Molecular Dynamics Using Stochastic Titration. J. Chem. Phys. 2002, 117, 4184-4200.

(56) Swails, J. M.; Roitberg, A. E. Enhancing Conformation and Protonation State Sampling of Hen Egg White Lysozyme Using $\mathrm{pH}$ Replica Exchange Molecular Dynamics. J. Chem. Theory Comput. 2012, 8, 4393-4404.

(57) Götz, A. W.; Williamson, M. J.; Xu, D.; Poole, D.; Le Grand, S.; Walker, R. C. Routine Microsecond Molecular Dynamics Simulations with AMBER on GPUs. 1. Generalized Born. J. Chem. Theory Comput. 2012, 8, 1542-1555.

(58) Xu, D.; Williamson, M. J.; Walker, R. C. Advancements in Molecular Dynamics Simulations of Biomolecules on Graphical Processing Units. In Annual Reports in Computational Chemistry; Wheeler, R. A. , Ed.; Elsevier: Amsterdam, 2010; Vol. 6, pp 2-19.

(59) Friedrichs, M. S.; Eastman, P.; Vaidyanathan, V.; Houston, M.; Legrand, S.; Beberg, A. L.; Ensign, D. L.; Bruns, C. M.; Pande, V. S. Accelerating Molecular Dynamic Simulation on Graphics Processing Units. J. Comput. Chem. 2009, 30, 864-872.

(60) Harvey, M. J.; Giupponi, G.; Fabritiis, G. D. ACEMD: Accelerating Biomolecular Dynamics in the Microsecond Time Scale. J. Chem. Theory Comput. 2009, 5, 1632-1639.

(61) Brown, W. M.; Wang, P.; Plimpton, S. J.; Tharrington, A. N. Implementing Molecular Dynamics on Hybrid High Performance Computers: Short Range Forces. Comput. Phys. Commun. 2011, 182, 898-911.

(62) Bergonzo, C.; Campbell, A. J.; de los Santos, C.; Grollman, A. P.; Simmerling, C. Energetic Preference of 8-oxoG Eversion Pathways in a DNA Glycosylase. J. Am. Chem. Soc. 2011, 133, 14504-14506.

(63) Shan, Y.; Seeliger, M. A.; Eastwood, M. P.; Frank, F.; Xu, H.; Jensen, M. Ø.; Dror, R. O.; Kuriyan, J.; Shaw, D. E. A Conserved Protonation-Dependent Switch Controls Drug Binding in the Abl Kinase. Proc. Natl. Acad. Sci. U.S.A. 2009, 106, 139-144.

(64) Qin, B. Y.; Bewley, M. C.; Creamer, L. K.; Baker, H. M.; Baker, E. N.; Jameson, G. B. Structural Basis of the Tanford Transition of Bovine B-Lactoglobulin. Biochemistry 1998, 37, 14014-14023.

(65) Case, D. A.; Darden, T. A.; Cheatham, T. E., III; Simmerling, C. L.; Wang, J.; Duke, R. E.; Luo, R.; Walker, R.C.; Zhang, W.; Merz, K.M., et al. AMBER 12; University of California: San Francisco, 2012.

(66) Hornak, V.; Abel, R.; Okur, A.; Strockbine, B.; Roitberg, A.; Simmerling, C. Comparison of Multiple Amber Force Fields and Development of Improved Protein Backbone Parameters. Proteins: Struct., Funct., Bioinf. 2006, 65, 712-725.

(67) Bikiel, D. E.; Boechi, L.; Capece, L.; Crespo, A.; Biase, P. M. D.; Lella, S. D.; Lebrero, M. C. G.; Martí, M. A.; Nadra, A. D.; Perissinotti, L. L.; et al. Modeling Heme Proteins Using Atomistic Simulations. Phys. Chem. Chem. Phys. 2006, 8, 5611-5628.

(68) Ryckaert, J.-P.; Ciccotti, G.; Berendsen, H. J. C. Numerical Integration of the Cartesian Equations of Motion of a System with Constraints: Molecular Dynamics of N-Alkanes. J. Comput. Phys. 1977, 23, 327-341.

(69) Sindhikara, D. J.; Kim, S.; Voter, A. F.; Roitberg, A. E. Bad Seeds Sprout Perilous Dynamics: Stochastic Thermostat Induced Trajectory Synchronization in Biomolecules. J. Chem. Theory Comput. 2009, 5, 1624-1631.

(70) Onufriev, A.; Bashford, D.; Case, D. A. Exploring Protein Native States and Large-Scale Conformational Changes with a Modified Generalized Born Model. Proteins: Struct., Funct., Bioinf. 2004, 55, 383-394.

(71) O'Neil, L. L.; Grossfield, A.; Wiest, O. Base Flipping of the Thymine Dimer in Duplex DNA. J. Phys. Chem. B 2007, 111, 1184311849. 